\title{
Temperature induced modification of the mid-infrared response of single-walled carbon nanotubes
}

\author{
Mikhail V. Shuba, ${ }^{1, a)}$ Alesia G. Paddubskaya, ${ }^{2}$ Polina P. Kuzhir, ${ }^{1}$ Sergey A. Maksimenko, ${ }^{1}$ \\ Gintaras Valusis, ${ }^{2}$ Nikolai A. Poklonski, ${ }^{3}$ Stefano Bellucci, ${ }^{4}$ George Kenanakis, ${ }^{5}$ \\ and Maria Kafesaki ${ }^{5}$ \\ ${ }^{1}$ Institute for Nuclear Problems, Belarus State University, Bobruiskaya 11, 220030 Minsk, Belarus \\ ${ }^{2}$ Center for Physical Sciences and Technology, A. Gostauto 11, Vilnius LT-01108, Lithuania \\ ${ }^{3}$ Department of Physics, Belarus State University, Nezalezhnastsi Avenue 4, 220030 Minsk, Belarus \\ ${ }^{4}$ Frascati National Laboratory, National Institute of Nuclear Physics, E. Fermi 40, 00044 Frascati, Italy \\ ${ }^{5}$ Institute of Electronic Structure and Laser, Foundation for Research and Technology Hellas, P.O. Box 1385, \\ 71110 Heraklion, Crete, Greece
}

(Received 4 November 2015; accepted 25 February 2016; published online 11 March 2016)

\begin{abstract}
The temperature dependences of the absorbance spectra of thin free-standing single-walled carbon nanotube (SWCNT) films were studied in the infrared range $\left(700-6200 \mathrm{~cm}^{-1}\right)$ while heating the air from 300 to $575 \mathrm{~K}$. The observed temperature variation in the infrared absorbance spectra has been explained by two different physical factors. The first one is the strong temperature dependence of the conductivity of p-type doped semiconducting SWCNTs. The second one is the temperature dependence of electron relaxation time of intraband electron transitions in metallic SWCNTs. The possibility of the separation of contributions from the interband and intraband transitions to the infrared spectra of SWCNT films has been demonstrated. (C) 2016 AIP Publishing LLC.

[http://dx.doi.org/10.1063/1.4943499]
\end{abstract}

\section{INTRODUCTION}

A single-wall carbon nanotube (SWCNT) can be considered a rolled-up hexagonal sheet of carbon atoms. ${ }^{1}$ Depending on its chirality, which is directly connected with direction of rolling, the tube is semiconducting or metallic. One-dimensional nanotube topology leads to a set of one-dimensional energy bands for electrons. Both intraband transitions and direct interband electron transitions between valence and conduction bands contribute to the infrared conductivity of SWCNT. ${ }^{2}$ The former provide quasi-free motion of $\pi$-electrons and determine the metallic properties of SWCNTs in the frequency range much below interband electron transitions. The latter cause resonant interaction of SWCNTs with near-infrared and visible radiation. There is a broad intermediate frequency range $(30-150 \mathrm{THz})$ where the contributions from intra- and interband transitions to nanotube conductivity can be comparable. The optical properties of SWCNT films in this intermediate range have been measured at various conditions: at different temperatures, ${ }^{3-7}$ external hydrostatic pressures, ${ }^{3}$ applied electric fields, ${ }^{3-8}$ and chemical dopings. ${ }^{6,9,10}$ However, the competitive contributions from intra- and interband transitions to the infrared absorbance spectra have not been separated and analyzed yet.

Due to their high aspect ratio and unique electronic properties, the SWCNTs have been proposed as nanoantennas and nanowaveguides for infrared range.,11-14 Since interband transitions suppress surface wave propagation in SWCNTs $^{11,15}$ and consequently diminish their potential in application as nanoantennas and waveguides, the separation

${ }^{\text {a)} E l e c t r o n i c ~ m a i l: ~ m i k h a i l . s h u b a @ g m a i l . c o m ~}$ of the contributions from inter- and intraband transitions to the infrared nanotube conductivity is an important task. Due to the difference in temperature dependence of intraband conductivity (which increases as temperature grows) and interband conductivity (which decreases as temperature grows), examining and measuring the temperature dependence of the absorbance is a simple and unambiguous way to identify the relative contribution of inter- and intraband transitions to the IR conductivity of the SWCNTs. Here we demonstrate separation of the contribution from intra- and interband transitions to the infrared response of SWCNT films based on the measurements of the absorbance spectra of both doped and undoped SWCNT films at different temperatures.

As was shown earlier, ${ }^{3-7}$ the infrared absorbance spectra of SWCNT films observed in the temperature range 50-300 K demonstrate a weak temperature dependence that was attributed to a tube-to-tube variation in the chemical potential. ${ }^{16}$ Theoretical description for the temperature dependence of the infrared absorption spectra of SWCNT films has been given in Ref. 16, taking into account contribution from quasi-metallic SWCNTs with curvature induced small energy gap $(\sim 20 \mathrm{meV})$. However, the contribution from large gap (0.3-1.2 eV) semiconducting SWCNTs has been eliminated from consideration in Ref. 16.

In the present paper, we demonstrate a sufficiently strong $(7 \%)$ temperature induced variation in the mid-infrared spectra of the absorbance (A) of free-standing SWCNT thin films during heating (or cooling) in air in the temperature range $300-575 \mathrm{~K}$. We show that this originates from the strong temperature dependence of the conductivity of p-type doped large-gap semiconducting SWCNTs. The temperature 
variation in the mid-infrared spectra is used to separate the contributions from different types of transitions to the absorbance spectra of SWCNT films in the infrared range $f / c$ $\in(700,6200) \mathrm{cm}^{-1}(f \in(21,186) \mathrm{THz}) ; f$ is frequency and $c$ is speed of light in vacuum.

\section{EXPERIMENTAL DETAILS}

In experiments, we used SWCNTs (CG100, SWeNT, Inc.) produced by chemical vapor deposition (CVD) on a cobalt-molybdenum catalyst, with an average diameter of $0.82 \mathrm{~nm}$, carbon purity exceeding $90 \%$, and SWCNT content of more than $75 \%$. As the diameter of the SWCNTs varies from 0.7 to $1.3 \mathrm{~nm}$, the electronic band gap of semiconducting SWCNTs lies in the range $0.7-1.3 \mathrm{eV}^{8}$ According to the equiprobability of SWCNTs of identical radii but different chirality, ${ }^{1}$ the ratio of the density of semiconducting tubes to the density of metallic tubes in used SWCNT material was assumed to equal 2.

Free standing $200-400 \mathrm{~nm}$ thin films comprising randomly oriented SWCNTs were prepared following Ref. 9 (for details, see supplementary materials ${ }^{17}$ ). Some of the films were doped while the others were annealed. In order to dope the SWCNT films with acid, they were put into $3 \mathrm{wt} . \%$ nitric acid for $5 \mathrm{~h}$ before being transferred onto a substrate. To anneal the SWCNT films, they were heated in a vacuum at $500^{\circ} \mathrm{C}$ for $1.5 \mathrm{~h}$. The annealing procedure was used to remove organic and non-organic molecules from the SWCNT surfaces. These non-organic molecules become attached to the SWCNT surface during SWCNT purification process in acid and can provoke doping effects. Organic surfactant molecules were attached to SWCNTs during their dispersion in the suspension at SWCNT film fabrication. After annealing and removing the SWCNT films from vacuum, the atmospheric oxygen attaches to the sidewall defects at SWCNT surface, providing a slight unintentional p-type doping effect. ${ }^{18}$

With normally incident light, the absorbance spectra were obtained in the infrared and visible ranges $700-6200 \mathrm{~cm}^{-1}$ and $8500-17000 \mathrm{~cm}^{-1}$ with a resolution of $1 \mathrm{~cm}^{-1}$, using a Fourier-transform infrared (FT-IR) spectrometer (Bruker Vertex 70v). Number of scan was 10 , and detectors were DTGS and Si-Diode for the ranges $700-6200 \mathrm{~cm}^{-1}$ and $8500-17000 \mathrm{~cm}^{-1}$, respectively.

We used the "Linkam FT-IR600 Heating and Cooling Stage" in order to heat the samples. The measurement of the spectra was conducted $3 \mathrm{~min}$ after temperature stabilization, and the samples were maintained at each fixed temperature value for $5 \mathrm{~min}$. Using this approach, we avoided significant degradation of the electronic properties of SWCNTs due to partial oxidation in the air at high temperatures (typically $>550 \mathrm{~K}$ ). As some surfactant remained in the SWCNT films, the mid-infrared spectra of the films had absorption bands associated with vibration of different bonds, including $\mathrm{C}-\mathrm{O}, \mathrm{C}=\mathrm{O}, \mathrm{O}-\mathrm{H}, \mathrm{C}-\mathrm{H}$, and $\mathrm{C}-\mathrm{C}$. As we were interested in the absorbance due to intrinsic IR conductivity of SWCNTs, we omitted these bands in our considerations and exclude them in the way demonstrated in Fig. S2 of supplementary material. ${ }^{17}$

\section{EXPERIMENTAL RESULTS AND DISCUSSION}

\section{A. Annealed thin film measurements}

The annealed free standing films were subjected to three heating-cooling cycles (to ensure and identify reversible and non-reversible changes): in the range 300-475 K (1st cycle), then in the range $300-525 \mathrm{~K}$ (2nd cycle), and finally in the range $300-575 \mathrm{~K}$ ( $3 \mathrm{rd}$ cycle). In order to avoid oxidation of the SWCNTs and demonstrate reversible changes to their conductivity with temperature variation, the upper heating temperature was permanently increased from cycle to cycle.

Fig. 1(a) presents the mid-IR absorbance spectra of annealed SWCNT thin films at $300 \mathrm{~K}\left(\mathrm{~A}_{300}\right), 425 \mathrm{~K}\left(\mathrm{~A}_{425}\right)$, and $575 \mathrm{~K}\left(\mathrm{~A}_{575}\right)$ for the cooling in the 3-rd cycle. The absorbance increases with increasing temperature in the low frequency part of the spectra $\left(f / c<2200 \mathrm{~cm}^{-1}\right)$, where the contribution from the intraband transitions (quasi-free electron excitation) prevails. In the region where the contribution from interband electron transitions to SWCNT conductivity dominates $\left(f / c>2200 \mathrm{~cm}^{-1}\right)$, the absorbance of the SWCNT film decreases as temperature grows.

The ratio of the absorbances $A_{575} / A_{300}$ measured at 575 and $300 \mathrm{~K}$ is presented in Fig. 1(b). One can see that the ratio tends to a constant value 1.07 and 0.975 in high and low frequency part of the spectra, where conductivity is determined mostly by intraband and interband transitions, respectively. Such behavior indicates that temperature growth leads to (i) the increase of the free-charge carrier concentration in valence band and (ii) the decrease of the concentration of the electrons which can be excited from valence to conduction band by the IR radiation.

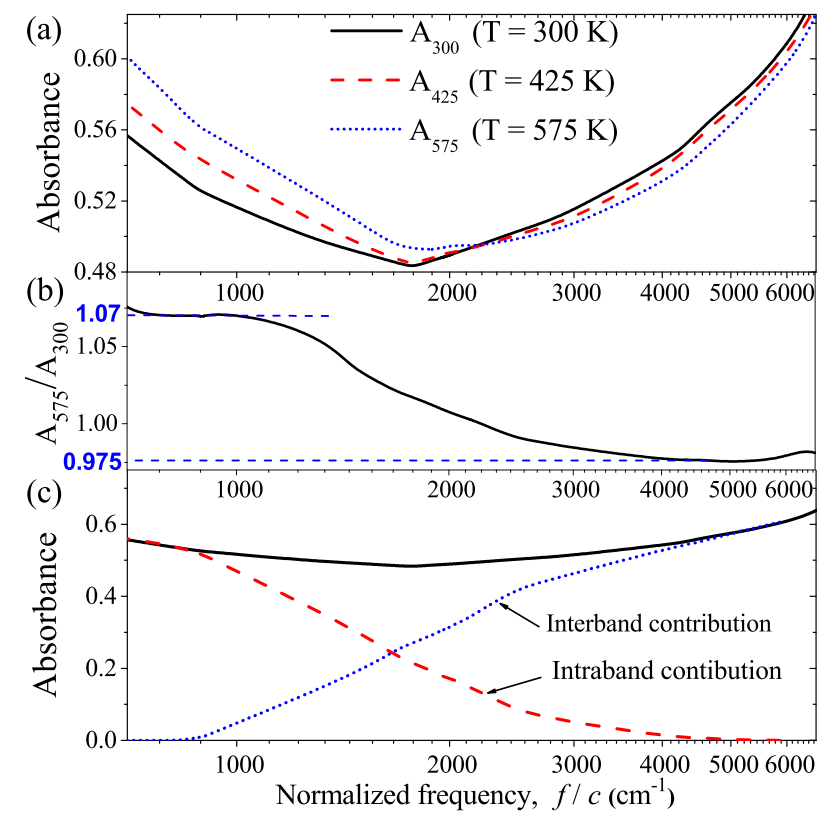

FIG. 1. (a) Infrared absorbance spectra A of thin, $400 \mathrm{~nm}$, free-standing SWCNT film at different temperatures, $300 \mathrm{~K}, 425 \mathrm{~K}$, and $575 \mathrm{~K}$. (b) The ratio of the absorbances $\mathrm{A}_{575} / \mathrm{A}_{300}$ presented in (a). The dashed lines indicate constant values corresponding to the frequency independence of the ratio $\mathrm{A}_{575} / \mathrm{A}_{300}$. (c) Infrared absorbance spectra of thin free-standing SWCNT film at $300 \mathrm{~K}$ (solid line) and evaluated contributions to this spectrum from intraband (dashed line) and interband (dotted line) transitions. 
The valence and conduction bands and dopant-induced levels located close to the top of valence band are schematically shown in Fig. 2. The electrons predominantly occupy the valence band at low temperature. As the temperature is raised, the electron thermal energy increases and some electrons are excited from the top of the valence band to the dopant-induced states. ${ }^{19,20}$ Consequently, dopant molecules on the SWCNT surface can attract electrons from the tube, resulting in the decrease of the number of interband transitions and increase of the number of intraband transitions. Thus, we can suppose that observed temperature dependence of the absorbance originates from the temperature dependence of the electron Fermi distribution function ${ }^{21}$

$$
F(E, T)=\left(1+\exp \left[(E-\mu) / k_{B} T\right]\right)^{-1},
$$

where $T$ is the temperature, $k_{B}$ is the Boltzmann constant, $E$ is the electron energy, and $\mu$ is the chemical potential. Note that for undoped tubes $\mu=0 \mathrm{eV} . F(E, T)$ strongly depends on the temperature if the Fermi level is located close to the top of valence band or to the bottom of conduction band, i.e., $|E-\mu|<5 k_{B} T$ and $|\mu|<E_{g} / 2$, where $E_{g}$ is the energy bandgap.

Let us also note that the temperature dependence of the electron relaxation time $\tau$ in SWCNT can lead to the temperature induced modification of the absorbance spectra of SWCNT film. We applied the theoretical model discussed in Section IV to calculate absorbance spectra of SWCNT film at two different $\tau$ and found that the variation in the electron relaxation time with temperature should lead to a slight frequency dependence of the value $\mathrm{A}_{575} / \mathrm{A}_{300}$; however, this dependence is not observed in our experiments in the range $[700,1000] \mathrm{cm}^{-1}$. Furthermore, variation in electron relaxation time with temperature is unable to explain (i) the very small temperature dependence of the absorbance spectra in the range from $2000-6200 \mathrm{~cm}^{-1}$ (see Fig. 1(a)) with inequality $d A / d T<0$ and (ii) the enhanced temperature dependence of the absorbance for doped films (see Sec. III B). Therefore, we suppose that the temperature dependence of electron relaxation time may contribute to the temperature-induced

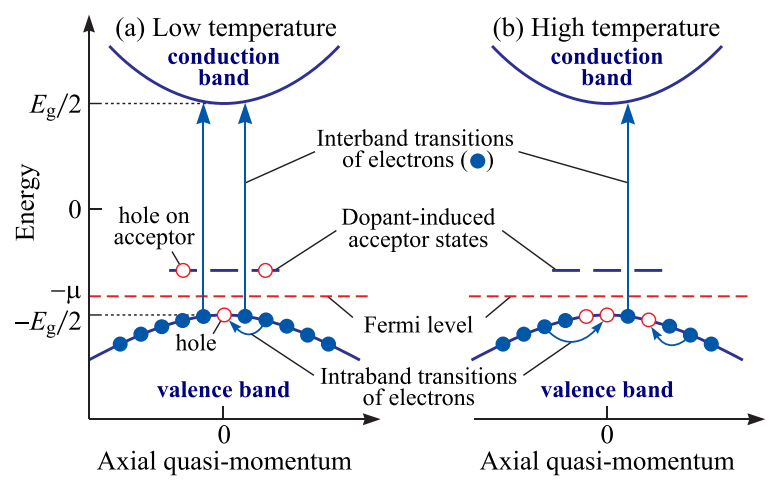

FIG. 2. The scheme of electron distributions over energy levels in p-type slightly doped semiconducting SWCNTs at (a) low and (b) high temperatures. Here $\mu$ is the chemical potential (Fermi level) and $E_{g}$ is the energy gap. When temperature rises, some holes leave the dopant-induced acceptor states going to the top of valence band. As a result, the number of interband transitions of electrons decreases while the number of intraband transitions increases. modification of intraband absorbance spectra of SWCNT film (see details in Sec. IV), but not explain it completely.

In the range $f / c>500 \mathrm{~cm}^{-1}$, the finite length effect is negligibly small for typical SWCNT lengths $(>1 \mu \mathrm{m})$, so that the conductivity of the film is proportional to the sum of SWCNT conductances in the unit volume. ${ }^{11}$ In this case, interband and intraband electron transitions contribute independently to the conductivity and to the absorbance of the SWCNT film allowing us to decompose the absorbance

$$
\mathrm{A}(T, f)=\mathrm{A}_{\text {inter }}(T, f)+\mathrm{A}_{\text {intra }}(T, f),
$$

where $\mathrm{A}_{\text {inter }}(T, f)$ and $\mathrm{A}_{\text {intra }}(T, f)$ are the contributions from inter- and intraband transitions, respectively. The decomposition (2) follows from Beer-Lambert law and it is usually applied to fit absorbance spectra by Drude-Lorentz formula. ${ }^{6}$ Eq. (2) is true when the film reflectance is small or one of the contributions is negligibly small; for example, this applies in the range $1000-6200 \mathrm{~cm}^{-1}$, where the reflectance is less than $4 \%$, and in the range $700-1000 \mathrm{~cm}^{-1}$, where $\mathrm{A}_{\text {inter }} \ll \mathrm{A}_{\text {intra }}$. From Fig. 1(b), we deduce the simple relation between contributions to the absorbance from intraband transitions at 300 and $575 \mathrm{~K}$

$$
\mathrm{A}_{\text {intra }}(575 \mathrm{~K}, f)=1.07 \mathrm{~A}_{\text {intra }}(300 \mathrm{~K}, f)
$$

and analogical relation for the contribution from interband transitions

$$
\mathrm{A}_{\text {inter }}(575 \mathrm{~K}, f)=0.975 \mathrm{~A}_{\text {inter }}(300 \mathrm{~K}, f) .
$$

Relations (3) and (4) are based on the assumption that absorbance changes with increasing temperature due to the temperature-induced variation in concentration of the electrons participating in interband and intraband transitions (see Fig. 2); as this variation is determined by Fermi function (1) and, consequently, does not depend on frequency, we supposed both these relations are true over the entire frequency range considered.

After substituting (3) and (4) into (2) and using (2) at two different temperatures, $300 \mathrm{~K}$ and $575 \mathrm{~K}$, we have a system of two linear equations allowing us to extract the contributions from the interband and intraband transitions to the absorbance of SWCNT film

$$
\left\{\begin{array}{l}
\mathrm{A}(300 \mathrm{~K})=\mathrm{A}_{\text {intra }}(300 \mathrm{~K})+\mathrm{A}_{\text {inter }}(300 \mathrm{~K}) \\
\mathrm{A}(575 \mathrm{~K})=1.07 \mathrm{~A}_{\text {intra }}(300 \mathrm{~K})+0.975 \mathrm{~A}_{\text {inter }}(300 \mathrm{~K})
\end{array}\right.
$$

Fig. 1(c) shows the contributions to the infrared absorbance spectrum from intraband and interband transitions of thin free-standing SWCNT film at $300 \mathrm{~K}$, calculated according to Eq. (5). As shown in Fig. 1(c), interband transitions are significant at wavenumbers higher than $900 \mathrm{~cm}^{-1}$, while intraband transitions strongly influence the spectra below $4000 \mathrm{~cm}^{-1}$. We obtained the same results using the separation technique analogically for the absorbance spectra at $300 \mathrm{~K}$ and another higher temperatures $T \in\{375,475$, $525\} \mathrm{K}$. This proves that the proposed separation method is an unambiguous and effective tool for analysis of the midinfrared absorption spectra of SWCNTs. Let us note that, in 
our approach, for the separation, it is not necessary to know the distribution of SWCNTs over diameters and chiral vectors.

As theoretically shown in Sec. IV, the effect of temperature dependence of relaxation time for electron intraband transition can lead to slight variation of the proportional coefficient in relation (3) as frequency varies, resulting in some error in the separation method. This problem does not appear for the separation method based on the measurement of the film absorbance spectra at the same temperature but different doping levels (see Section III B).

The reproducibility of the absorbance variation with temperature during three heating-cooling cycles is clearly seen in Figs. 3(a) and 3(b) for $f / c=700 \mathrm{~cm}^{-1}$ and $4700 \mathrm{~cm}^{-1}$, respectively. Some irreversible changes occur at $4700 \mathrm{~cm}^{-1}$ with temperatures higher than $525 \mathrm{~K}$. These changes can be associated with the partial oxidation of the SWCNTs leading to ptype doping and consequently a shift of the Fermi level closer to the valence band. As the heating of the air does not produce irreversible changes in the regime of intraband transitions, we can conclude that this oxidation-type doping is not strong and does not change the concentration of free-charge carries in SWCNTs.

Figure 4 shows visible and near-infrared absorbance spectra of the annealed SWCNT film measured at 575, 475, and $375 \mathrm{~K}$ during the sample cooling. The absorbance spectra have temperature dependence resembling that of the first interband transition resonance $\left(8000-10000 \mathrm{~cm}^{-1}\right)$ of large gap semiconducting SWCNTs. The tail of this resonance spreads into low frequency range up to $1000 \mathrm{~cm}^{-1}$ (see the long tail $\left(1000-8000 \mathrm{~cm}^{-1}\right)$ of the imaginary part of semiconducting tube conductivity in Fig. 7), so that semiconducting tubes determine the interband contribution to the nearinfrared absorbance of our samples.

\section{B. p-type doped thin film measurements}

Figures 5(a) and 5(b) present the temperature dependence of the absorbance of the doped SWCNT thin film during three heating-cooling cycles in the regimes of intraband $\left(700 \mathrm{~cm}^{-1}\right)$ and interband $\left(4700 \mathrm{~cm}^{-1}\right)$ electron transitions.
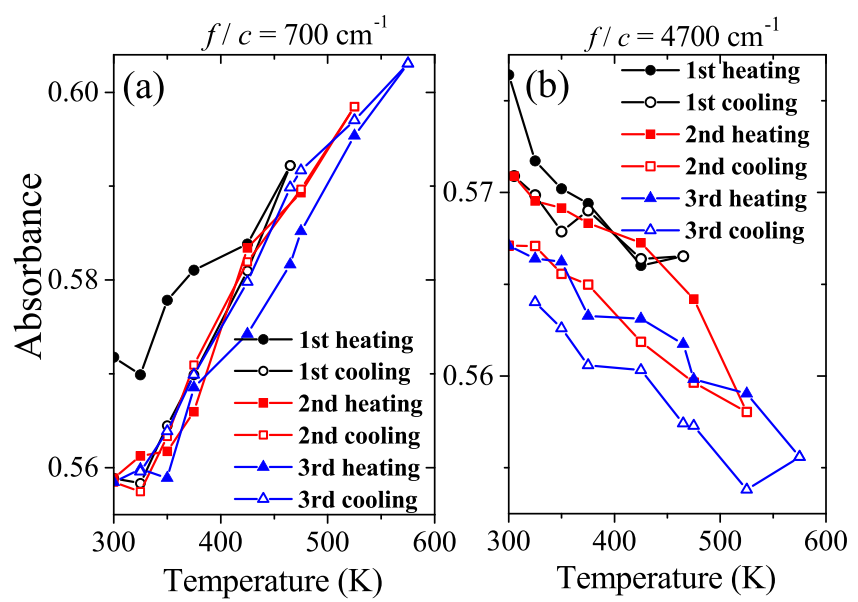

FIG. 3. Absorbance of annealed thin free-standing SWCNT film at different temperature during three heating and cooling cycles at $700 \mathrm{~cm}^{-1}$ (a) and $4700 \mathrm{~cm}^{-1}$ (b).

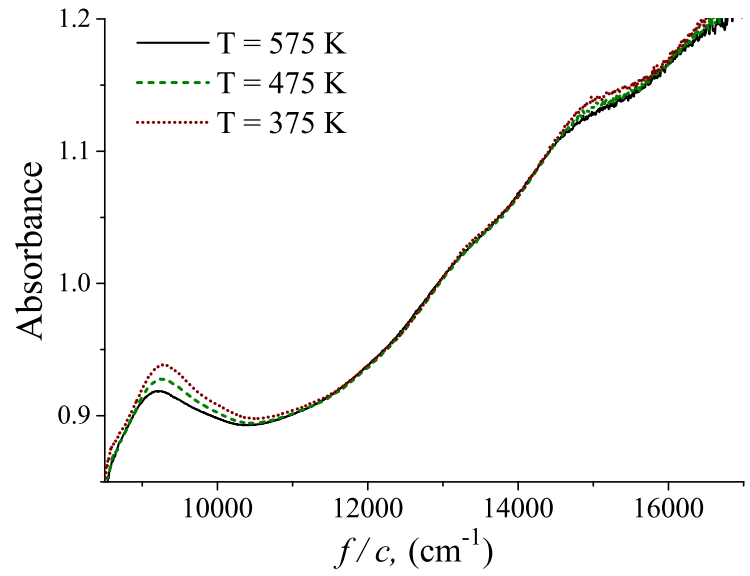

FIG. 4. Absorbance spectra of annealed free-standing SWCNT film at $375 \mathrm{~K}, 475 \mathrm{~K}$, and $575 \mathrm{~K}$.

As Fig. 5(c) demonstrates, the reversible changes of the absorbance spectra with temperature variation for a doped film are qualitatively the same as for an annealed (undoped) film, shown in Fig. 1(a). However, the temperature dependence of the absorbance both in the interband and intraband transition regimes is stronger in the case of a doped film (compare Figs. 3 and 5). This fact indicates that large-gap semiconducting tubes provoke strong temperature dependence of SWCNT film conductivity, as conductivity of other types of SWCNTs is not affected by the doping effect. The irreversible changes of the absorbance, caused by undoping and doping processes, occur at temperatures higher than $425 \mathrm{~K}$ at the first heating and higher than $475 \mathrm{~K}$ at the second and the third heating (see Figs. 5(a) and 5(b)). The first heating up to $525 \mathrm{~K}$ leads to the partial loss of the complexes, which remain on the tube after the acid treatment and cause doping effect. During the second heating (see Figs. 5(a) and 5(b)) at $525 \mathrm{~K}$, the undoping, simultaneously with oxidation, leads to irreversible reduction of the absorbance in both intraband and interband regimes.

Let us compare the absorbance spectra of the doped film before and after irreversible changes appeared during the
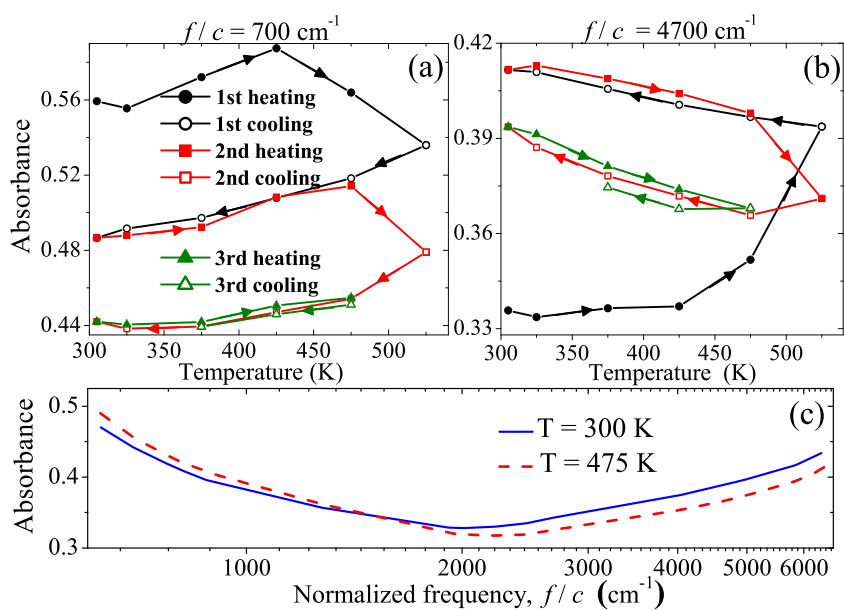

FIG. 5. Absorbance of doped SWCNT film at different temperature during three heating and cooling cycles at normalized frequencies $700 \mathrm{~cm}^{-1}$ (a) and $4700 \mathrm{~cm}^{-1}$ (b). (c) Infrared absorbance spectra of doped SWCNT film at $300 \mathrm{~K}$ and $475 \mathrm{~K}$ of the 3 rd cycle. 


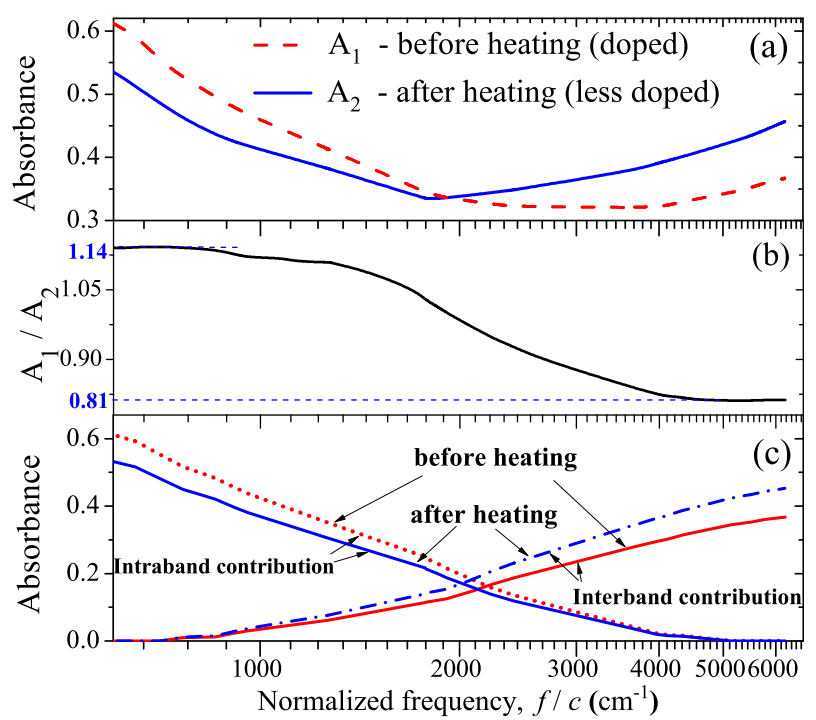

FIG. 6. (a) Infrared absorbance spectra of doped thin free-standing SWCNT film before $\left(A_{1}\right)$ and after $\left(A_{2}\right)$ heating at $475 \mathrm{~K}$ for $5 \mathrm{~min}$. The spectra were measured at $375 \mathrm{~K}$. (b) The ratio of the absorbances $A_{2} / A_{2}$ presented in (a). The dashed lines indicate constant values corresponding to the frequency independence of the ratio $A_{1} / A_{2}$. (c) The contributions of interband and intraband transitions to the absorbance of doped thin free-standing SWCNT film presented in (a) before and after heating.

first heating-cooling cycle. The spectra of the doped film at $375 \mathrm{~K}$ before $\left(\mathrm{A}_{1}\right)$ and after $\left(\mathrm{A}_{2}\right)$ the first heating-cooling cycle are shown in Fig. 6(a). The spectrum of ratio $A_{1} / A_{2}$ in Fig. 6(b) is identical to the spectrum of the ratio of absorbance of annealed film at different temperatures presented in Fig. 1(b). This indicates that irreversible undoping process leads to a decrease in the concentration of the free electrons and an increase in the concentration of bound electrons, which can be excited by the infrared radiation. Following the method presented above (see Eqs. (2)-(5)), we separated the contributions from inter- and intraband transitions to the absorbance of the doped film before and after the first heatingcooling cycle (see Fig. 6(c)). Fig. 6(c) demonstrates spectra of interband and intraband absorbance before and after the heating induced undoping process. Thus, the interband and intraband contributions to the absorbances of SWCNT film can be separated if one uses the absorbance spectra of SWCNT film at two slightly different doping extents.

\section{THEORETICAL CONSIDERATION AND DISCUSSION}

Semi-classical Drude-Lorentz dielectric function model is usually applied to describe absorption spectra of SWCNTs. ${ }^{6}$ However, this model does not contain any direct temperature dependence of the parameters of Drude-Lorentz formula. That is why, here, in order to describe and explain the main tendencies observed in our experiments, we applied the quantum transport theory based approach.

In our experiment, the samples were mixtures of both metallic and semiconducting SWCNTs. The conductivity of the undoped metallic $S W C N T s$ with small radius $(<2.3 \mathrm{~nm})$ follows the Drude law, where plasma frequency does not depend on the temperature. ${ }^{2}$ The same is true for doped metallic tubes. As density of electron states for metallic tubes is constant in the range between the first van Hove singularities, the shift of the Fermi level within this range cannot change the conductivity of metallic SWCNTs. However, electron relaxation time of intraband transitions may have temperature dependence and may cause the dependence of metallic tube conductivity on the temperature. However, this cannot completely explain the observed temperature dependence of our samples in the mid-infrared range.

We suppose that the observed reversible temperature induced variation of SWCNT conductivity occurs due to the doping of semiconducting SWCNTs by oxygen molecules ${ }^{18}$ for annealed samples and by nitric acid complexes ${ }^{22,23}$ for doped samples. In order to prove our supposition, we calculated conductivity spectra of doped SWCNTs and absorbance spectra of SWCNT-based composites in the infrared range at different temperatures. As all the semiconducting tubes have qualitatively the same infrared response, we did our calculation just for one type of SWCNTs. Based on the quantum transport theory (see supplementary material ${ }^{17}$ ), we computed the spectra of the axial surface conductivity of doped semiconducting "zigzag" SWCNT with chiral indexes $(10,0)$ at different temperatures, 300 and $525 \mathrm{~K}$. The electron relaxation time was taken as 20 and 40 fs for inter- and intraband transitions, respectively; ${ }^{11}$ they were assumed to be constant in the considered temperature interval. The energy gap of the chosen tube was $E_{g}=1.064 \mathrm{eV}$. The chemical potential of the tube was taken to be $0.47 \mathrm{eV}$ so that the Fermi level locates close to the top of the valence band (see Fig. 2), and the energy difference between the top of valence band and the Fermi level is comparable with the energy of thermal motion: $E_{g} / 2-|\mu|=2.5 k_{B} T_{r}, T_{r}=300 \mathrm{~K}$ is a room temperature. In this case, thermal motion can influence the electron population of the valence band: when the temperature increases, some electrons leave the valence band resulting in an increase of the intraband transitions and a decrease of interband transitions (see Fig. 2).

In general, both chemical potential $\mu$ and energy gap $E_{g}$ depend on the temperature, so that $d|\mu| / d T \leq 0$ (see Ref. 21) or $d|\mu| / d T>0$ and $d E_{g} / d T \leq 0$ (see Ref. 24). The decrease of both $\mu$ and $E_{g}$ with the sample heating should lead to an increase of absorption in the range of interband transitions, but the opposite was observed in our experiments. As such, dependences $\mu(T)$ and $E_{g}(T)$ can only weaken (but not determine) the observed temperature dependence of the absorbance spectra in the range $1000-6000 \mathrm{~cm}^{-1}$; in our calculations for simplicity, we supposed $\mu$ and $E_{g}$ to be constant in the considered temperature range, from $300 \mathrm{~K}$ to $525 \mathrm{~K}$.

Fig. 7 demonstrates the calculated conductivity spectra for doped semiconducting "zigzag" SWCNT at different temperatures. As shown in Fig. 7, the low-frequency conductivity (regime of intraband transitions: $400-1000 \mathrm{~cm}^{-1}$ ) increases more than 4 times as the temperature increases from 300 to $525 \mathrm{~K}$. In the high frequency part of the spectra in Fig. 7 (the regime of the first interband transition: $\left.2000-10000 \mathrm{~cm}^{-1}\right)$, the conductivity decreases by about $15 \%$ with increasing temperature. The calculations show that the relative temperature-induced variation of both interband and intraband conductivities of semiconducting SWCNTs 
does not depend on frequency; this justifies application of the relations 3 and 4 .

Following Ref. 11, the relative effective permittivity or dielectric constant of a film containing the randomly oriented SWCNTs

$$
\varepsilon_{e f f}=\varepsilon_{h}+i \sum_{j} \frac{2 \pi n_{j} R_{j} L_{j} \sigma_{j}}{3 \omega \varepsilon_{0}}
$$

where $\varepsilon_{h}$ is the relative permittivity of the host medium $\left(\varepsilon_{h}=1\right.$ for vacuum), index $j$ indicates type of the tube with specific chirality, $R_{j}, L_{j}$, and $\sigma_{j}$ are length, radius, and axial surface conductivity of $j$-type of SWCNT, respectively, and $n_{j}$ is the nanotube number density which relates with the nanotube volume fraction $f_{j}$, as $f_{j}=\pi R_{j}^{2} L_{j} n_{j}$.

Using (6) and the Fresnel formulas for the transmission and reflection by planar slabs, we calculated the absorbance of a thin model film comprised of identically doped semiconducting SWCNTs, with conductivity presented in Fig. 7, and identical metallic SWCNTs with chiral indices $(12,0)$. To be specific, a 1:2 ratio between the fraction of metallic tubes and the fraction of semiconducting tubes was assumed. In order to show how the temperature dependence of electron Fermi distribution function $F(T)$ results in the temperatureinduced modifications of the film absorbance spectra, we assumed relaxation time of intraband transitions to be constant in considered temperature range $(\tau(T)=40 \mathrm{ps})$; in this approximation, the conductivity of metallic tubes does not depend on temperature. ${ }^{2}$ The results for absorbance and the ratio of absorbances $A_{300}$ and $A_{525}$ at $300 \mathrm{~K}$ and $525 \mathrm{~K}$ are presented in Figs. 8 (a) and 8(b), respectively. The absorbance ratios $A_{525} / A_{300}$ calculated at the two different temperatures have qualitatively the same frequency behavior as in the experiment (compare Figs. 1(b) and. 8(b)).

In order to demonstrate how metallic tubes can contribute to the temperature dependence of the film absorbance, we assumed that relaxation time of intraband transitions decreased from $40 \mathrm{fs}$ to $35 \mathrm{fs}$ as temperature increased from $300 \mathrm{~K}$ to $525 \mathrm{~K}$. The absorbance of the model film $A_{525}^{\prime}$ at $525 \mathrm{~K}$ and $\tau=35 \mathrm{ps}$, and the ratio of absorbances $A_{525}^{\prime} / A_{300}$ are presented in Figs. 8(a) and 8(b). The difference between $A_{525}$ and $A_{525}^{\prime}$ in Fig. 8(a) demonstrates the contribution of metallic tubes to the temperature dependence of the film absorbance. The conductivity of both metallic and

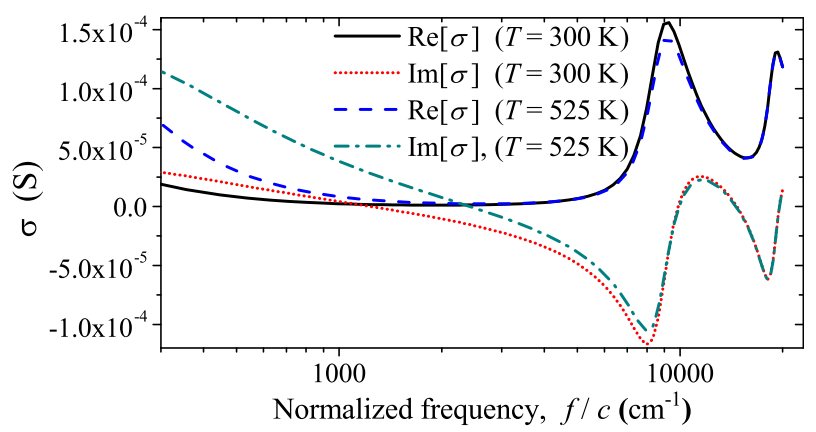

FIG. 7. Calculated axial surface conductivity $\sigma$ of the semiconducting SWCNT $(10,0)$ with the energy bandgap $1.064 \mathrm{eV}$ and chemical potential $\mu=0.467 \mathrm{eV}$ at 300 and $525 \mathrm{~K}$.
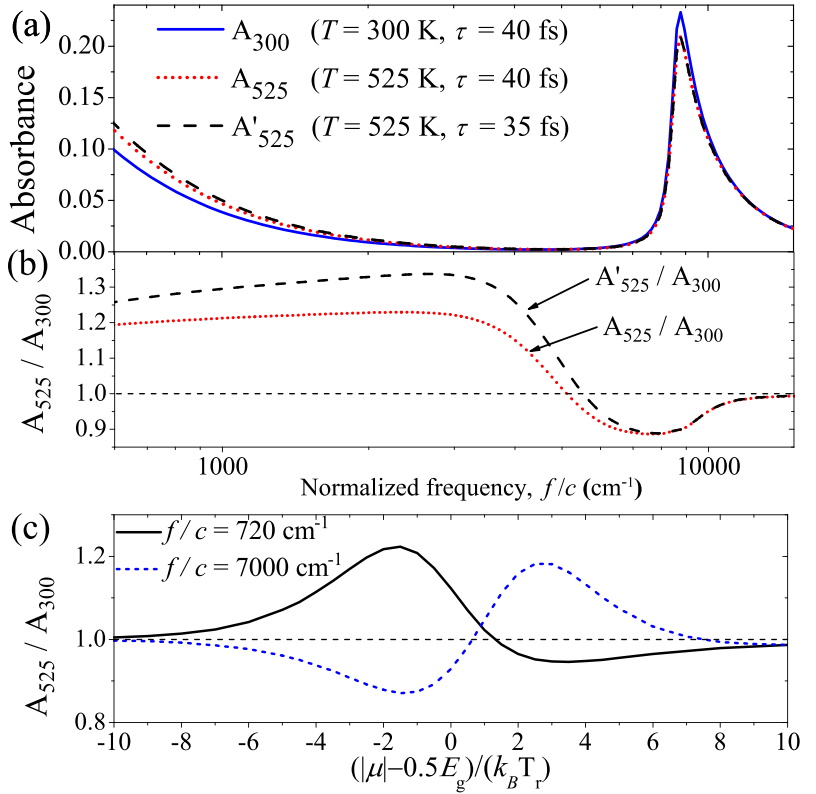

FIG. 8. (a) The calculated absorbance spectra of thin films comprised of identically doped semiconducting SWCNTs $(10,0)$ and identical metallic SWCNTs $(12,0)$ at $525 \mathrm{~K}\left(A_{525}\right)$ and $300 \mathrm{~K}\left(A_{300}\right)$ at the same intraband relaxation time $\tau=40 \mathrm{fs}$; additionally, absorbance at $525 \mathrm{~K}\left(A_{525}^{\prime}\right)$ was calculated at $\tau=35 \mathrm{fs}$. The conductivity of the semiconducting SWCNTs was taken to be the same as in Fig. 7. The volume fraction of the tubes was $2 \%$ and the film thickness was $200 \mathrm{~nm}$. Tube length was $1 \mu \mathrm{m}$. (b) The ratios of the absorbances $A_{525} / A_{300}$ and $A_{525}^{\prime} / A_{300}$ presented in (a). (c) The ratio of the absorbances $A_{525} / A_{300}$ versus $\left(|\mu|-0.5 E_{g}\right) / k_{B} T_{r}$ at normalized frequencies $720 \mathrm{~cm}^{-1}$ and $7000 \mathrm{~cm}^{-1}$. The value $\left(|\mu|-0.5 E_{g}\right) / k_{B} T_{r}$ is the normalized energy difference between the Fermi energy and the top of the valence band of the doped semiconducting SWCNT; here $E_{g}=1.064 \mathrm{eV}$ and $T_{r}=300 \mathrm{~K}$.

semiconducting tubes increases in the regime of intraband transition as temperature increases. However, different types of tubes provide different frequency dependences of temperature induced relative absorbance variations:

(i) An increase of the number of intraband transitions in semiconducting tubes with the sample heating leads to frequency independence of the ratio of semiconducting tube conductivities at different temperatures $T_{1}$ and $T_{2}$, i.e., $\left|\sigma_{s}\left(T_{1}, f\right)\right| /\left|\sigma_{s}\left(T_{2}, f\right)\right|=$ const is true in the regime of intraband transitions. This is demonstrated in Fig. 8(b), where $A_{525} / A_{300}$ is almost constant in the range below $2000 \mathrm{~cm}^{-1}$; small frequency dependence of the $A_{525} / A_{300}$ in Fig. 8(b) can be explained by the reflection, which becomes stronger at smaller frequencies.

(ii) A decrease of the electron relaxation time in metallic SWCNTs leads to slight frequency dependence of the ratio of metallic tube conductivities at different temperatures, i.e., $\left|\sigma_{m}\left(T_{1}, f\right)\right| /\left|\sigma_{m}\left(T_{2}, f\right)\right| \neq$ const. This is demonstrated in Fig. 8(b), where $A_{525}^{\prime} / A_{300}$ has slight frequency dependence in the range below $2000 \mathrm{~cm}^{-1}$. The contribution of metallic SWCNT to the temperature dependence of film absorbance spectra can lead to partial break of equality (3), resulting in some error in the separation method applied in Sec. III A.

Thus, we conclude that the observed temperature variation of SWCNT film absorbance spectra in the mid-infrared 
range could be explained by two effects: (i) the strong temperature dependence of the conductivity of p-type doped semiconducting SWCNTs and (ii) the temperature dependence of electron relaxation time of intraband transitions in metallic SWCNTs. The first effect is significant in the whole mid-infrared range, whereas the second one could contribute only to the regime of intraband transitions below $4000 \mathrm{~cm}^{-1}$.

In order to appreciate how temperature induced variation of the film absorbance depends on the chemical potential $\mu$ of the chosen semiconducting tubes, we calculated the ratio of the absorbances $A_{525} / A_{300}$ versus $\left(|\mu|-0.5 E_{g}\right) /\left(k_{B} T_{r}\right)$ at $525 \mathrm{~K}$ and $300 \mathrm{~K}$ at two normalized frequencies $720 \mathrm{~cm}^{-1}$ (intraband transition regime) and $7000 \mathrm{~cm}^{-1}$ (interband transition regime) (see Fig. 8(c)). The value $\left(|\mu|-0.5 E_{g}\right) /$ $\left(k_{B} T_{r}\right)$ is the normalized energy difference between the Fermi level and the top of the valence band for the chosen SWCNT with energy gap $E_{g}=1.064 \mathrm{eV} ; T_{r}=300 \mathrm{~K}$ is a room temperature. As shown in Fig. 8(c), all the tubes with Fermi level located in the range $|\mu| \in\left[0.5 E_{g}-8 k_{B} T_{r}, 0.5 E_{g}\right.$ $+0.5 k_{B} T_{r}$ ] contribute significantly in the same way as was demonstrated in our experiments: i.e., SWCNT film absorbance increases (decreases) with increasing temperature in the intraband (interband) transition regime.

However, as follows from Fig. 8(c), for heavier doped SWCNTs with the Fermi level located inside valence band $\left(|\mu| \in\left(0.5 E_{g}+0.5 k_{B} T_{r}, 0.5 E_{g}+7 k_{B} T_{r}\right)\right)$ the temperature dependence of absorbance spectra is opposite to what we observed in the experiment. The explanation of the increase of interband absorbance (at $7000 \mathrm{~cm}^{-1}$ ) and the decrease of intraband absorbance (at $720 \mathrm{~cm}^{-1}$ ) with increasing temperature for this doping level is as follows. At low temperature, $T=300 \mathrm{~K}$, the electrons locate in the vicinity of the Fermi level and participate both in inter- and intraband transitions (see Fig. 9(a)). At higher temperature, $T=525 \mathrm{~K}$, some electrons occupy states above Fermi level, i.e., electron energy is $E \in\left(-|\mu|,-E_{g} / 2\right)$ (see Fig. 9(b)). Those electrons (i) participate in the interband transitions at smaller frequencies, this leads to red shift of interband transition resonance;

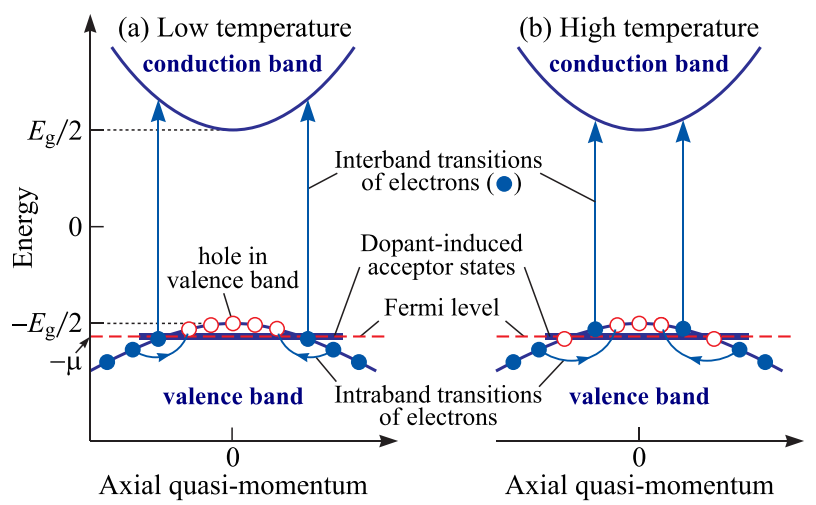

FIG. 9. The scheme of electron distributions over energy levels in p-type heavily doped semiconducting SWCNTs at (a) low and (b) high temperatures. Fermi level is located inside valence band so that $|\mu|-E_{g} / 2 \approx 2 k_{B} T_{r}$, $T_{r}=300 \mathrm{~K}$. When temperature rises, some electrons occupy states above the Fermi level; they participate in interband transitions at smaller frequencies and do not participate in intraband transitions in the mid-infrared range. As a result, the number of intraband transitions decreases, and a red shift of interband transition resonance occurs. (ii) do not participate in the intraband transitions as photon energy at $720 \mathrm{~cm}^{-1}$ is higher than the energy of possible intraband transitions i.e., $2 \pi f \hbar>E_{g} / 2-|E|$.

The contributions of both intra- and interband transitions to the absorbance spectra of heavily doped semiconducting SWCNTs practically do not depend on the temperature in the mid-infrared range (see Fig. 8(c) at $\left(|\mu|-0.5 E_{g} / 2\right) /$ $\left.\left(k_{B} T_{r}\right)>8\right)$. This is due to complete "metallization" of semiconducting SWCNTs; their mid-infrared conductivity does not depend on the temperature, in analogy to that of metallic SWCNTs. ${ }^{2}$ The last statement is true if the temperature dependence of the electron relaxation time in SWCNTs is negligibly small.

\section{CONCLUSION}

In conclusion, we observed a temperature variation $(2 \%-7 \%)$ in the absorbance of free-standing SWCNT films (both annealed and doped) in the infrared range $\left(700-6200 \mathrm{~cm}^{-1}\right)$ while heating from 300 to $575 \mathrm{~K}$. The absorbance spectra of doped samples demonstrate stronger temperature dependence than annealed ones. Based on the quantum mechanical theory of SWCNT conductivity, we demonstrated that the temperature variation of the spectra can be explained by two factors: (i) the strong temperature dependence of the conductivity of p-type doped semiconducting SWCNTs, and (ii) the temperature dependence of electron relaxation time of intraband electron transitions in metallic SWCNTs. We also showed it is possible to separate the interband and intraband contributions to the infrared spectra of SWCNT film: the method is based on the measurement of absorbance spectra of SWCNTs film either at two different temperatures or at two different doping extents. The method can be useful for interpreting the midinfrared spectra of SWCNT films.

\section{ACKNOWLEDGMENTS}

The work was financially supported by the Belarusian Republican Foundation for Fundamental Research (BRFFR) under Project Nos. F14R-026, F14V-003, EU FP7 under Project Nos. FP7-612285 CANTOR, PIRSES-GA-2012318617, FP7-318617 FAEMCAR, FP7-316633 POCAONTAS.

${ }^{1}$ S. Reich, C. Thomsen, and J. Maultzsch, Carbon Nanotubes. Basic Concepts and Physical Properties (Wiley-VCH, Berlin, 2004).

${ }^{2}$ G. Y. Slepyan, S. A. Maksimenko, A. Lakhtakia, O. Yevtushenko, and A. V. Gusakov, Phys. Rev. B 60, 17136 (1999).

${ }^{3}$ K. Thirunavukkuarasu, F. Hennrich, K. Kamaras, and C. A. Kuntscher, Phys. Rev. B 81, 045424 (2010).

${ }^{4}$ A. Ugawa, A. G. Rinzler, and D. B. Tanner, Phys. Rev. B 60, R11305 (1999).

${ }^{5}$ A. Ugawa, J. Hwang, H. Gommans, H. Tashiro, A. Rinzler, and D. Tanner, Curr. Appl. Phys. 1, 45 (2001).

${ }^{6}$ F. Borondics, K. Kamarás, M. Nikolou, D. B. Tanner, Z. H. Chen, and A. G. Rinzler, Phys. Rev. B 74, 045431 (2006).

${ }^{7}$ Q. Zhang, E. H. Haroz, Z. Jin, L. Ren, X. Wang, R. S. Arvidson, A. Luttge, and J. Kono, Nano Lett. 13, 5991 (2013).

${ }^{8}$ F. Wang, M. E. Itkis, and R. C. Haddon, Nano Lett. 10, 937 (2010).

${ }^{9}$ Z. Wu, Z. Chen, X. Du, J. M. Logan, J. Sippel, M. Nikolou, K. Kamaras, J. R. Reynolds, D. B. Tanner, A. F. Hebard, and A. G. Rinzler, Science 305, 1273 (2004). 
${ }^{10}$ K. Kamaras, A. Pekker, B. Botka, H. Hu, S. Niyogi, M. E. Itkis, and R. C. Haddon, Phys. Status Solidi B 247, 2754 (2010).

${ }^{11}$ G. Y. Slepyan, M. V. Shuba, S. A. Maksimenko, C. Thomsen, and A. Lakhtakia, Phys. Rev. B 81, 205423 (2010).

${ }^{12}$ G. Hanson, IEEE Trans. Antennas Propag. 53, 3426 (2005).

${ }^{13}$ P. J. Burke, S. Li, and Z. Yu, IEEE Trans. Nanotechnol. 5, 314 (2006).

${ }^{14}$ G. Y. Slepyan, M. V. Shuba, S. A. Maksimenko, and A. Lakhtakia, Phys. Rev. B 73, 195416 (2006).

${ }^{15}$ M. V. Shuba, G. Y. Slepyan, S. A. Maksimenko, C. Thomsen, and A. Lakhtakia, Phys. Rev. B 79, 155403 (2009).

${ }^{16}$ T. Kampfrath, K. von Volkmann, C. M. Aguirre, P. Desjardins, R. Martel, M. Krenz, C. Frischkorn, M. Wolf, and L. Perfetti, Phys. Rev. Lett. 101, 267403 (2008)

${ }^{17}$ See supplementary material at http://dx.doi.org/10.1063/1.4943499 for details of SWCNT film preparation; an example of the subtraction of narrow absorption bands from the spectra; formulas for SWCNT axial surface conductivity.

${ }^{18}$ P. G. Collins, K. Bradley, M. Ishigami, and A. Zettl, Science 287, 1801 (2000).

${ }^{19}$ C. Zhou, J. Kong, and H. Dai, Appl. Phys. Lett. 76, 1597 (2000).

${ }^{20}$ J. Kong, N. R. Franklin, C. Zhou, M. G. Chapline, S. Peng, K. Cho, and H. Dai, Science 287, 622 (2000).

${ }^{21}$ C. Kittel and H. Kroemer, Thermal Physics (W. H. Freeman, New York, 1980).

${ }^{22}$ R. Graupner, J. Abraham, A. Vencelova, T. Seyller, F. Hennrich, M. M. Kappes, A. Hirsch, and L. Ley, Phys. Chem. Chem. Phys. 5, 5472 (2003).

${ }^{23}$ F. Hennrich, R. Wellmann, S. Malik, S. Lebedkin, and M. M. Kappes, Phys. Chem. Chem. Phys. 5, 178 (2003).

${ }^{24}$ R. B. Capaz, C. D. Spataru, P. Tangney, M. L. Cohen, and S. G. Louie, Phys. Rev. Lett. 94, 036801 (2005). 